\title{
IMPURITY TRANSPORT AND RADIATION
}

\author{
M.Z. Tokar and M. Koltunov \\ Institute for Energy and Climate Research - Plasma Physics, Forschungszentrum Jülich GmbH, \\ EURATOM Association, Trilateral Euregio Cluster, D-52425 Jülich, Germany
}

\begin{abstract}
The role of impurity radiation at the plasma edge in fusion devices is considered. Conditions critical for radiative instabilities, provoking the development of detachment and MARFE, are analyzed. Requirements necessary for the creation of a stable radiating edge, that would allow to protect the wall from intensive heat loads, are formulated.
\end{abstract}

\section{INTRODUCTION}

From the very beginning of nuclear fusion research an uncontrollable pollution of the plasma core with impurities released through the interaction of hot particles with the wall was a subject of deep concerns. It was early recognized that radiation losses from such impurities can hinder the plasma heating to thermonuclear temperatures and the concentrations, being "lethal" for fusion, have been estimated for different impurity species $[1,2]$.

Experiments on earlier tokamak devices with wall elements of heavy metals, e.g., stainless steel, molybdenum, tungsten, have demonstrated that instead of a gradual increase of radiation e.g., with increasing plasma density, the losses can start to grow explosively when the density exceeds a certain critical level (see, e.g., [3, 4]). This behavior is caused by a sudden accumulation of heavy impurity particles of high charges near the plasma axis. This fact forced researchers to switch to light elements like carbon, beryllium, boron in fabricating the wall facing components. The ions of these elements radiate most intensively at relatively low temperatures, i.e., in the edge region close to the plasma boundary [5]. This edge radiation can play a positive role reducing heat loads concentrated mostly on particular wall elements, such as limiters and divertor target plates $[6,7]$. Nevertheless, also in this case the plasma behavior does not obey simple laws. The radiating edge layer attached to the plasma boundary can become unstable when the plasma density is ramped up above a threshold value [3]. Under some conditions this manifests itself in a radial contraction of the plasma column that preserves its poloidal and toroidal homogeneity $[3,9,10]$. By such a detachment the power launched into the plasma is completely lost with impurity radiation from a thin toroidal shell at the edge. Often a detachment terminates the discharge through disruption [11] but may also lead to the formation of a quasi-stationary "detached plasma". In other cases a toroidal plasma loop of very high density and low temperature, named the Multi-Faceted Radiation From the Edge (MARFE), arises at the high field side of the device and later can disappear or smear out into a "detached plasma" [12, 13, 14]. In divertor machines the processes of MARFE formation is initiated often by a plasma detachment from neutralizing plates and MARFE locates near the X-point [15].

A compromise has been found by deliberate seeding into the plasma of impurities of intermediate atomic numbers like neon, silicon, argon $[6,7]$. On the one hand, their characteristics allow to increase the edge radiation to a level of $95 \%$ of the power transported from the plasma core without formation of MARFE or "detached plasmas" [7]. On the other hand, their electric charge is still low enough to avoid accumulation in the plasma core. Such a "radiating edge" allows to reduce significantly the heat flows to the wall elements.

Further exploration of the "radiating edge" concept has resulted in one of the most unexpected findings in controlled fusion research: under definite condition impurity seeding results in a reduction of anomalous heat and particle losses from the plasma $[16,17]$. These discovery has shown that impurities are essentially involved not only in the global plasma behavior but also in the mechanisms of anomalous transport induced by micro-instabilities developing at very small spatial scales. The Radiation Improved (RI) mode, a regime with the energy confinement increased by the presence of impurities, is now considered as an attractive scenario for a fusion reactor. This mode combines the benefits both from the reduction of head loads on material surfaces and from improved energy confinement in the plasma core.

This brief introduction gives an idea about the broadness of the spectrum of impurity impacts on hot plasmas in fusion devices. These impacts extend from the negative phenomenon of radiation instability leading to MARFE and "detached plasma" to a desirable improvement of confinement in the RI-mode. Investigations of mechanisms underlying impurity influence pursue both academic and practical aims: (i) 
deeper insight into radiative phenomena, (ii) better understanding of impurity involvement in transport processes and (iii) outgrowth of a coherent understanding of the impurity behavior in hot plasmas in order to optimize this in future reactors.

In the present contribution only the processes important from the point of view of radiation losses from impurities and their impact on the thermal stability at the plasma edge in fusion plasmas are considered.

\section{POWER DENSITY OF RADIATION LOSSES}

Normally, impurity enters the plasma as neutral particles, generated in erosion processes of the wall elements $[18,19]$ or deliberately seeded $[7,8]$. In the process of ionization by electrons these neutrals are converted into ions of different electric charges $Z$. The power density of radiation losses from all impurity charge states can be calculated as follows:

$$
Q_{\mathrm{rad}}=\sum_{Z=0}^{Z_{\max }} n n_{Z} L_{Z}
$$

Here $n$ is the density of plasma electrons which loss their energy either by exciting electrons bounded in impurity ions or due to Bremsstrahlung radiation by Coulomb scattering; $n_{Z}$ the density of impurity ions of the charge $Z ; L_{Z}$ the so called cooling rate, i.e., the power lost from a unit volume if one electron and one impurity ion are present there. To determine $n_{Z}$ and $L_{Z}$, one has to consider the processes responsible for the transport of impurity particles and radiation from them.

If the exact spreading of each impurity charge state is non-relevant, one can express $Q_{\text {rad }}$ through the effective impurity cooling rate $L_{I}=\sum_{Z} \zeta_{Z} L_{Z}$ and the total impurity ion density $n_{I}=\sum_{Z} n_{Z}$ :

$$
Q_{\mathrm{rad}}=n n_{I} L_{I}
$$

where $\zeta_{Z}=n_{Z} / n_{I}$ are the relative concentrations of different impurity charge states.

\section{A. Impurity ion density}

The densities of ions of different charges are described by the continuity equations:

$$
\partial_{t} n_{Z}+\nabla_{\|}\left(n_{Z} V_{\|}^{Z}\right)+\nabla_{\perp} \Gamma_{\perp}^{Z}=S_{Z}-\nu_{Z} n_{Z}
$$

where $V_{\|}^{Z}$ is the ion velocity along magnetic field, $\Gamma_{\perp}^{Z}$ the density of their flux in the perpendicular direction, $S_{Z}$ and $\nu_{Z}$ are the source density and the frequency of ion disintegration, respectively. The latter include diverse processes [20], e.g., ionization by electrons, capture of free electrons by radiative and dielectronic recombination, and of electrons bounded in hydrogen atoms by charge-exchange, etc.

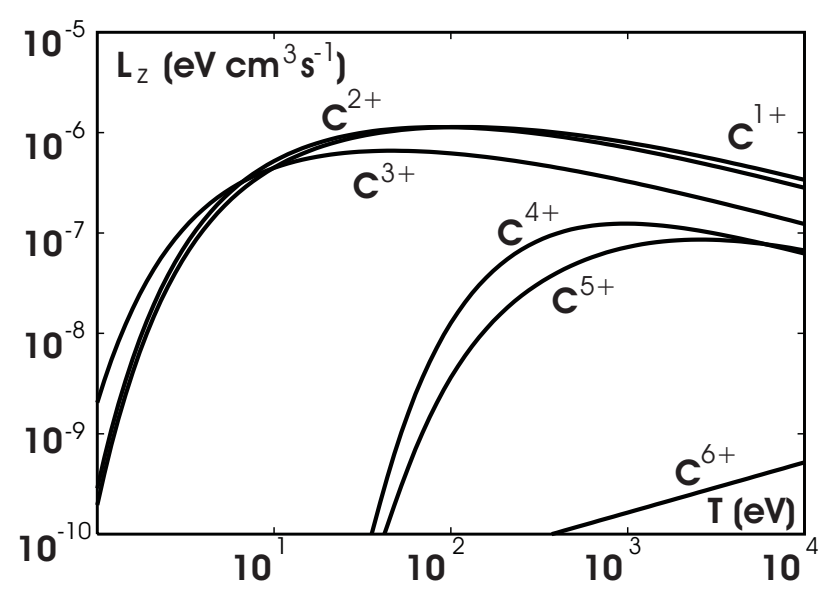

Figure 1: The temperature dependence of the cooling rates for different carbon charge states.

The parallel motion of impurity ions is of the most importance close to the sources where impurity particles are released into the plasma and obeys the momentum transfer equation:

$$
\begin{aligned}
& \partial_{t}\left(n_{Z} V_{\|}^{Z}\right)+\nabla_{\perp}\left(\Gamma_{\perp}^{Z} V_{\|}^{Z}\right) \\
& +\nabla_{\|}\left[n_{Z}\left(V_{\|}^{Z}\right)^{2}+n_{Z} T_{Z} / m_{Z}\right] \\
& =M_{Z}-\nu_{Z} n_{Z} V_{\|}^{Z}+Z e E_{\|} / m_{Z} \\
& +\nu_{Z i}\left(V_{\|}-V_{\|}^{Z}\right)+\xi_{Z} \nabla_{\|} T / m_{Z}
\end{aligned}
$$

The first term on the right hand side, $M_{Z}$, is the momentum assimilated from the ion source and the second one is the loss by the ion disintegration; the third term is the acceleration due to the parallel electric field; the forth one term is due to friction of impurities with the background ions moving with the mass parallel velocity $V_{\|}$, and $\nu_{Z i}$ is the friction coefficient [21]; the last term is the so called thermal force arising also due to collisions with the background plasma particles if their temperature $T$ has a parallel gradient; this force exists because the collision frequency decreases with increasing temperature and for the impurity ion mass $m_{Z}$ significantly larger than that of the background ions, $m_{i}, \xi_{Z} \approx 3.3 Z^{2}$ [22]. Normally, the electric field arises because light electrons escape from the plasma to the limiter or divertor plate faster than ions. In such a case both electric and friction forces drag the impurity ions back to the material surface but the thermal force pulls them in the opposite direction, towards the region of higher temperature.

The perpendicular flux of impurities, averaged over the magnetic surfaces, is normally decomposed into diffusive and convective terms:

$$
\Gamma_{Z \perp}=-D_{\perp} \nabla_{\perp} n_{Z}+V_{\perp} n_{Z}
$$

In the diffusivity $D_{\perp}$ and convection velocity $V_{\perp}$ usually neoclassical and anomalous contributions are 


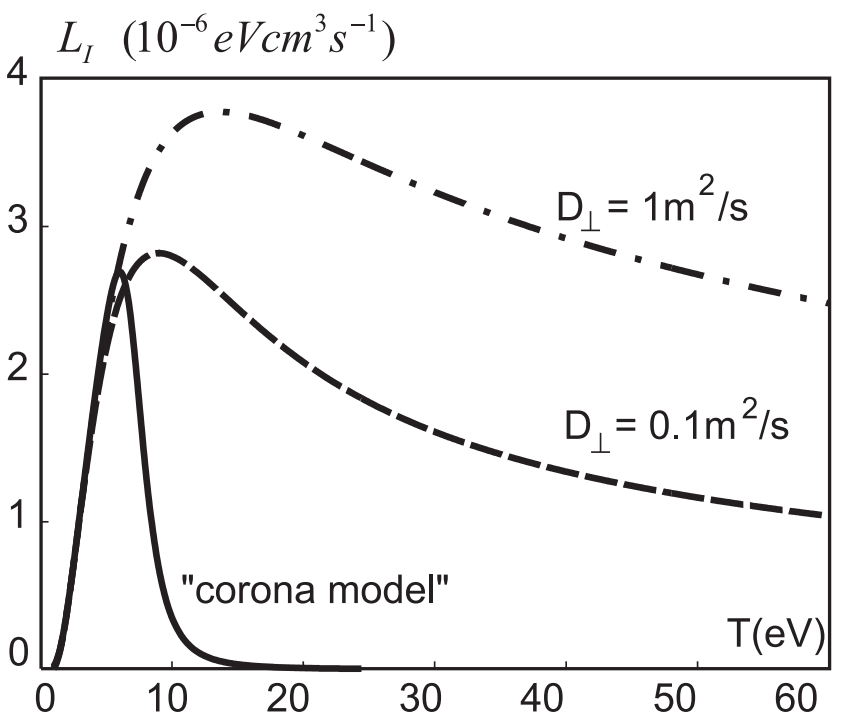

Figure 2: The effect of perpendicular diffusion of impurity ions on the effective cooling rate of carbon.

taken into account:

$$
D_{\perp}\left(V_{\perp}\right)=D_{\perp}^{\text {neo }}\left(V_{\perp}^{\text {neo }}\right)+D_{\perp}^{\text {an }}\left(V_{\perp}^{\text {an }}\right)
$$

The former one is due to collisions with the main ions [23] and the latter one - due to drift microinstabilities in the plasma [24]. Normally $D_{\perp}^{\text {neo }} \ll$ $D_{\perp}^{\text {an }}$ and $V_{\perp}^{\text {neo }} \lesssim V_{\perp}^{\text {an }}$. The convection velocities are controlled by the radial gradients of plasma parameters such as density, temperature, safety factor, etc.

The temperature $T_{Z}$ of impurity species in the parallel pressure gradient on the left hand side of Eq. (4) is changing through the Coulomb collisions with the background plasma particles and is governed by the heat transfer equation:

$$
\begin{aligned}
& \partial_{t}\left(1.5 n_{Z} T_{Z}\right)+\nabla_{\perp}\left(1.5 \Gamma_{\perp}^{Z} T_{Z}\right) \\
& +\nabla_{\|}\left(2.5 n_{Z} V_{\|}^{Z} T_{Z}\right) \\
& =Q_{Z}-1.5 \nu_{Z} n_{Z} T_{Z}+3 \nu_{Z i} n_{Z}\left(T-T_{Z}\right)
\end{aligned}
$$

where $Q_{Z}$ is the density of heat assimilated from the ion source. It is worth to note that different impurity charge states are heated by the plasma particles to different temperatures and in the vicinity of local sources of impurity the effect of impurity heating on its parallel transport can be comparable or even higher than that from the electric field arising by impurity ionization.

\section{B. Impurity cooling rate}

There are two the most important radiation processes through which plasma electrons lose their energy in interactions with impurity particles. The first one is the line radiation arising when impurity is excited by electron impacts [5]. In hot fusion plasmas the excited particles are normally deexcited spontaneously by radiating photons. Since
Power density of carbon radiation $\left(W / \mathrm{cm}^{3}\right)$

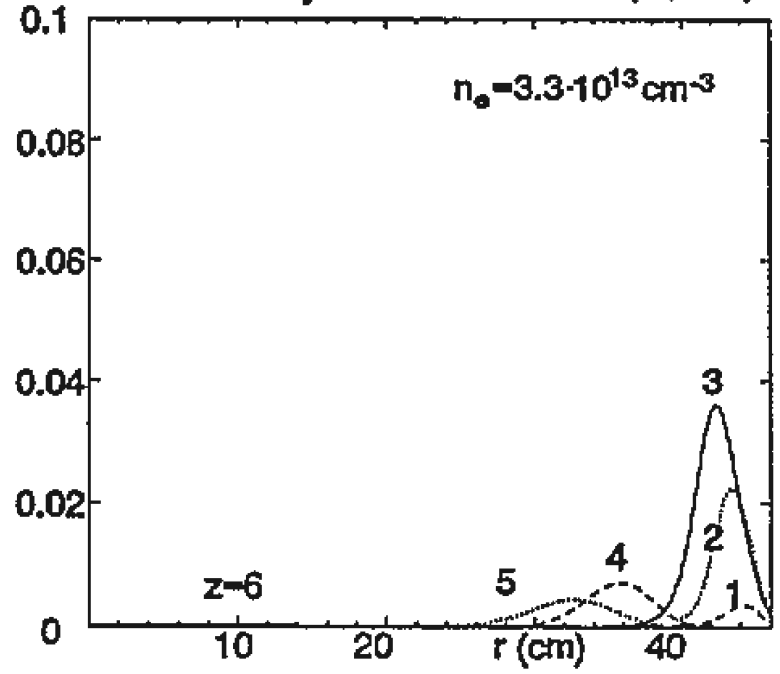

Figure 3: The radial profiles of the radiation loss density for different charge states of carbon impurity entering the plasma through the LCMS located at the minor radius $r=46 \mathrm{~cm}$, computed with the code RITM for an Ohmic discharge in the tokamak TEXTOR [27].

tokamak plasmas are normally transparent to impurity radiation, this leads to plasma cooling. The second one is Bremsstrahlung arising due to change of electron velocity caused by the attraction towards the impurity nuclei. Normally at the plasma edge the line radiation is the main contribution to the radiation from impurities. The cross-section of the Bremsstrahlung process increases, however, as $Z^{2}$ and in the hot central plasma, where impurity particles are strongly ionized, it dominates radiation losses. The temperature dependence of the cooling rate for carbon ions of all charges is shown in Fig. 1. The low ionized $\mathrm{B}, \mathrm{Be}$, Li-like charge states with $Z=1,2,3$ are easy to excite since their typical excitation energy $E_{\text {ex }}$ is of $5-10 \mathrm{eV}$; the He, H-like ions $\mathrm{C}^{4+}$ and $\mathrm{C}^{5+}$ with $E_{\text {ex }} \sim E_{\text {ion }} \gtrsim 300 \mathrm{eV}$ can be excited only at high temperatures; the nuclei $C^{6+}$ contribute to Bremsstrahlung only.

Often, e.g. in the hot central plasma, the effective impurity cooling rate $L_{I}$ is assessed in the so called corona approximation [5]. It has been developed for the description of Sun corona where the processes of ionization and recombination dominate the particle balances for different charge states and their densities are governed by the relations:

$$
k_{Z-1}^{\mathrm{ion}} n_{Z-1}+k_{Z+1}^{\mathrm{rec}} n_{Z+1}=\left(k_{Z}^{\mathrm{ion}}+k_{Z}^{\mathrm{rec}}\right) n_{Z}
$$

Here $k_{Z, Z \pm 1}^{\mathrm{ion}}$ and $k_{Z, Z \pm 1}^{\mathrm{rec}}$ are the ionization and recombination rate coefficients depending only on the electron temperature. In this case the relative concentrations of different impurity charge states, $\zeta_{Z}$, and, thus, the effective impurity cooling rate $L_{I}$ depend also on the local electron temperature only. This de- 
pendence is shown for carbon by the solid curve in Fig. 2. The increase of $L_{I}$ with the temperature at low $T$ is due to temperature behavior of $L_{Z}$ for impurity ions of low charges with small excitation energies, see Fig. 1. The sharp drop of $L_{I}$ at high temperatures is due to ionization of impurity particles into dim high-Z states.

At the plasma edge in fusion devices the anomalous transport can be very intensive. Low-Z impurity ions, which are generated from neutrals entering the plasma, have enough time to diffuse into hot plasma regions before they are ionized into dim high-Z states. Therefore, transport processes increase effective cooling rate and make it less temperature sensitive compared to the corona approximation without transport effects; see Fig. 2 where $L_{I}$ is shown by dashed and dash-dotted curves for different values of the impurity diffusivity $D_{\perp}[25]$. Similarly the charge-exchange of impurity ions with hydrogen neutrals affects $L_{I}$ [26]. The effects of elementary and transport processes on the density, radiation losses and ion heat exchange from impurity are taken firmly into account in transport codes. Figure 3 shows the radial profiles of the radiation loss density for different charge states of carbon computed by the code RITM (Radiation of Impurity and Transport Model) for an Ohmic discharge in the tokamak TEXTOR [27]. One can see that the Li-like ions $C^{3+}$ are the main contributors to the radiation losses. This is explained by the fact that, on the one hand, the characteristic excitation energy of these ions is relatively low, of $8 \mathrm{eV}$, and, on the other hand due to high enough ionization energy of $64 \mathrm{eV}$ they live long and penetrate deeply into the plasma.

In the vicinity of intense localized impurity sources, e.g. diagnostic beams, injection valves or suddenly melted parts of the wall, one has to take into account the essential time-dependence and threedimensionality of the impurity spreading process. During the life time $\tau_{\text {ion }}^{Z} \equiv 1 /\left(k_{Z}^{\text {ion }} n\right)$ impurity ions of the charge state $Z$ move along the magnetic field and diffuse in the direction $y$ perpendicular to the field at distances $l_{Z} \approx V_{\|}^{Z} \tau_{\text {ion }}^{Z}$ and $\delta_{Z} \approx \sqrt{D_{\perp} \tau_{\text {ion }}^{Z}}$, respectively. On the one hand the area $A_{Z}=\delta_{Z} l_{Z}$ occupied by ions of low $Z$ ions with very large $k_{Z}^{\text {ion }}$ and small $\tau_{\text {ion }}^{Z}$ is negligible compared with the total area $A_{S}$ of the magnetic surface. On the other hand, $k_{Z}^{\text {ion }}$ decreases with increasing $Z$ and, thus, $A_{Z}$ grows up also. Therefore, the $Z$-state serves as an intensive but localized source for the next charge state. All together the regions on the magnetic surface occupied by charge states with $A_{Z}<A_{S}$ form a set of nested shells evolving in time. By using this "shell" approximation [28] one can complement transport codes, modeling the radial profiles of impurity ion densities, e.g., RITM, with a description of non-stationary spreading of impurity on magnetic surfaces.

\section{THERMAL INSTABILITIES DUE TO RADIA- TION AND COULOMB COLLISIONS WITH IM- PURITY}

Presence of impurity can make a significant effect on the plasma parameters. In turn, this changes impurity transfer in such a perturbed plasma. The plasma can be significantly disturbed by: (i) the production of additional electrons by impurity ionization, (ii) electron heat losses on impurity excitation and ionization, (iii) heat transfer from the main ions to impurity ones by Coulomb collisions. The influence on the temperatures of electron and the main ion, $T_{e}$ and $T_{i}$, respectively, is governed by the heat transport equations:

$$
\begin{array}{r}
1.5 n \partial_{t} T_{e}-\kappa_{\|}^{e} \Delta_{\|} T_{e}-\kappa_{\perp}^{e} \Delta_{\perp} T_{e}=-Q_{\mathrm{rad}} \\
1.5 n \partial_{t} T_{i}-\kappa_{\|}^{i} \Delta_{\|} T_{i}-\kappa_{\perp}^{i} \Delta_{\perp} T_{i}=-Q_{\mathrm{coll}}
\end{array}
$$

where $\kappa_{\|}^{e} \gg \kappa_{\|}^{i} \gg \kappa_{\perp}^{e}>\kappa_{\perp}^{i}$ are the components of the electron and ion heat conductions parallel and perpendicular to the magnetic field and $Q_{\text {coll }}=3 \nu_{Z i} n_{Z}\left(T_{i}-T_{Z}\right)$ is the power density of heat losses due to Coulomb collisions [21]. Consider the plasma region inside the last closed magnetic surface (LCMS) in a limiter tokamak or inside the separatrix in a divertor device. Initially the temperatures $T_{e}=T_{i}=T_{0}$ are assumed constant on a magnetic surface and impurity ions are cold, $T_{Z} \ll T_{0}$. Consider a spontaneous fluctuation in the temperature periodic in the direction $l$ of the magnetic field:

$$
T_{e, i}=T_{0}+\delta T_{e, i} \times \exp (i k l+\gamma t)
$$

where $k=2 \pi / \lambda$ with $\lambda$ being the perturbation wave length, and $\gamma$ is the growth rate of the perturbation which has to be determined. The plasma pressure $P=n\left(T_{e}+T_{i}\right)$ is equilibrated along field lines very fast and the temperature change leads to the perturbation in the plasma density:

$$
\frac{\delta n}{n_{0}}=-\frac{\delta T_{e}+\delta T_{i}}{T_{0}}
$$

Thus, at the position where the temperature is reduced the density is increased. We on purpose omit here the finiteness of the time required for movement of the main ions to cooled area as it allows us to exclude acoustic waves inessential for this consideration. By using the latter relation and linearizing Eqs. (9) and (10) for small perturbations, we get a system of equations for the perturbation amplitudes $\delta T_{e, i}$ :

$$
\begin{gathered}
\gamma\left(2 \delta T_{e}+0.5 \delta T_{i}\right)+\frac{k^{2} \kappa_{\|}^{e}}{n} \delta T_{e} \\
=n_{I}\left[\frac{L_{I}}{2 T_{0}} \delta T_{i}+\left(\frac{L_{I}}{2 T_{0}}-\frac{d L_{I}}{d T_{0}}\right) \delta T_{e}\right] \\
\gamma\left(2 \delta T_{i}+0.5 \delta T_{e}\right)+\frac{k^{2} \kappa_{\|}^{i}}{n} \delta T_{i} \\
=\frac{Q_{\text {coll }}}{T_{0}}\left(\delta T_{i}+0.5 \delta T_{e}\right)
\end{gathered}
$$


which nontrivial solution provides an equation for perturbation growth rate. Consider two limit cases. If radiative electron cooling prevails, $\delta T_{e} \gg \delta T_{i}$, it reduces to:

$$
\gamma=\frac{n_{I}}{2}\left(\frac{L_{I}}{2 T_{0}}-\frac{d L_{I}}{d T_{0}}\right)-\frac{k^{2} \kappa_{\|}^{e}}{2 n}
$$

In the limit case of dominant Coulomb ion cooling, $\delta T_{i} \gg \delta T_{e}$, it provides:

$$
\gamma=\frac{Q_{\text {coll }}}{T_{0}}-\frac{k^{2} \kappa_{\|}^{i}}{n}
$$

Small spontaneous reduction of plasma component temperatures provokes an increase in plasma density, $n$, caused by the pressure equilibration. This increases the heat losses both from electrons through the rising radiation and from the main ions due to heat exchange with impurity ions in Coulomb collisions. As it is seen from Eqs. (14) and (15), in both cases plasma heat conduction reduces the growth rate of perturbation and plays a stabilizing role. An instability develops, i.e. $\gamma \geq 0$ and initial spontaneous perturbations grow exponentially with time, if with increasing either plasma density or impurity content the heat losses from the main plasma components exceed the critical level which can not be compensated by the heat fluxes coming to impurity cloud with heat conduction. This level can be described by the parameter $\eta=n_{I} n / k^{2}$. For electron radiation instability this threshold value is equal to:

$$
\eta_{\mathrm{rad}}=\frac{\kappa_{\|}^{e}}{0.5 L_{I} / T_{0}-d L_{I} / d T_{0}}
$$

and for cooling instability induced by heat exchange with impurity ions by Coulomb collisions:

$$
\eta_{\text {coll }}=\frac{\kappa_{\|}^{i} T_{0}}{Q_{\text {coll }}}
$$

This provides a hint for understanding why the MARFE, considered often as the non-linear stage of the radiation instability, develops in the inner plasma edge at the high field side (HFS). Due to the Shafranov shift of the plasma axis the distance between magnetic surfaces is larger at the HFS than at the low field side (LFS). Therefore, the radial temperature gradient and heat flux from the plasma core are weaker at the HFS. Already in the stationary state before plasma becomes unstable there is an inhomogeneity along the magnetic field so that the plasma temperature is somewhat lower and density is larger at the HFS than at the LFS. Therefore, the critical heat losses are reached first at the HFS and the radiation instability, leading to the MARFE formation, develops first there. One has to mention that also other mechanisms for the energy loss are of importance for the MARFE formation. In particular the perpendicular convection of charged particle from the MARFE

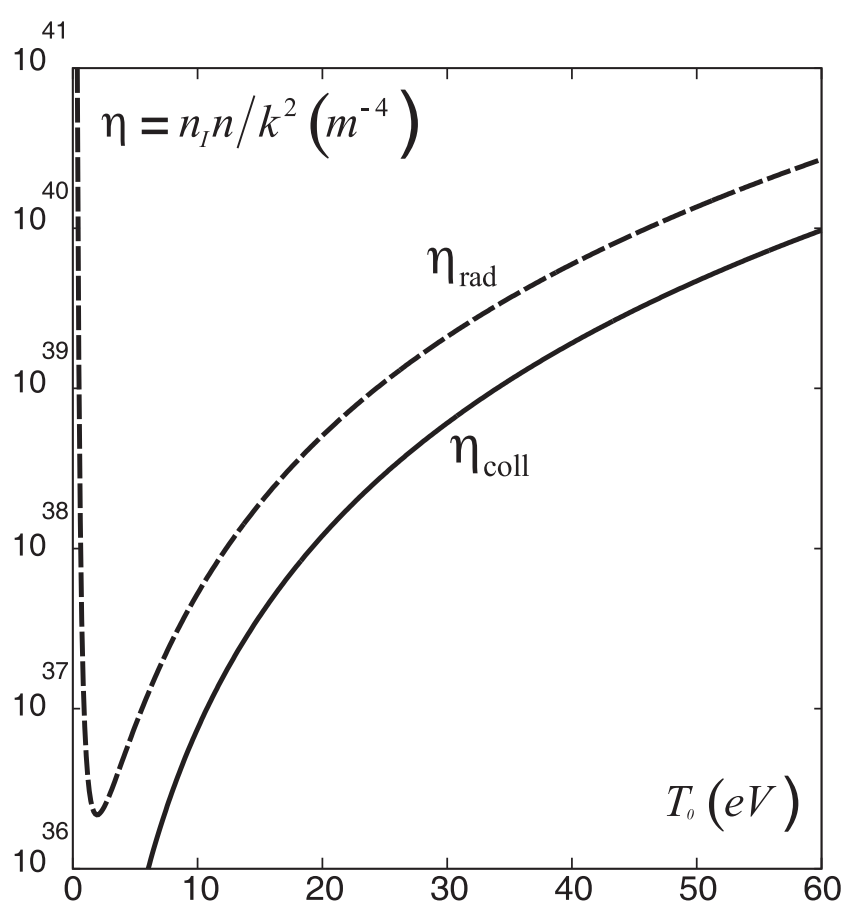

Figure 4: Critical parameter $\eta$ for the development of thermal instabilities due electron cooling with carbon radiation and cooling of the main ions by Coulomb collisions with impurities.

region with increased density to the inner wall is considered now as an important one [25, 30].

For particular plasma conditions and impurity species $\eta_{\text {rad }}$ and $\eta_{\text {coll }}$ are functions of initial plasma temperature $T_{0}$ only. Figure 4 demonstrates this dependences for typical parameters of deuterium plasma with carbon impurities [29]. One can see that for the conditions usual for MARFE development, with the edge temperature below $50 \mathrm{eV}$, ion collision instability can develop at the product of the plasma and impurity densities several times smaller, than that required for development of electron radiation instability. For higher charge states this difference even stronger since $Q_{\text {coll }} \sim \nu_{Z i} \sim Z^{2}$. Thus, by studying the MARFE development one has to take ion-ion collisions into account.

\section{STABLE RADIATING LAYER AT THE PLASMA EDGE}

Up to now we have neglected the fact that in fusion devices the temperature is inhomogeneous in the radial direction: since the heat is deposited into the plasma core and is transferred to the plasma edge with perpendicular plasma heat conduction a strong radial temperature gradient exists. This is, however, of very importance for the creation of a stable radiating layer at the edge. Such a layer would be very beneficial for the protection of wall elements from intensive heat loads and has been successfully realized in 


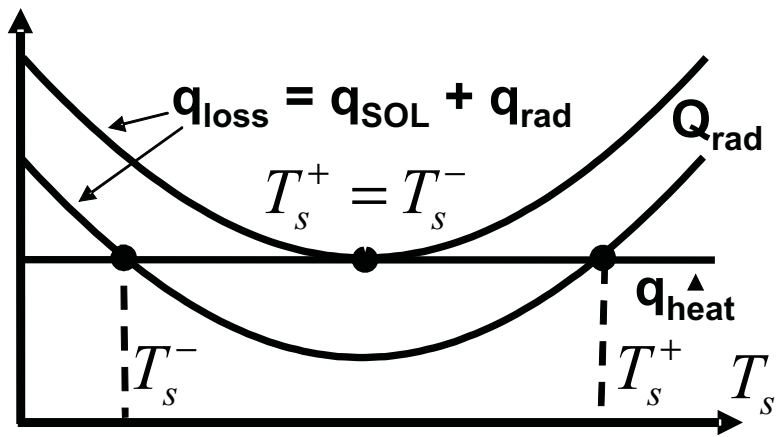

Figure 5: Dependence of the total losses from the plasma with the heat conduction to the SOL and edge radiation from impurity on the plasma temperature at the LCMS. Two stationary states can exist and that with the lower temperature is unstable. These states are merging, $T_{s}^{+}=T_{s}^{-}$, when the maximum radiation level is achieved. No stationary states exist when $q_{\text {heat }}$ is smaller than the minimum $q_{\text {loss }}$.

additionally heated discharges in TEXTOR by puffing of neon. Under these conditions up to $85-90 \%$ of the input power is radiated from the plasma edge inside the LCMS without MARFE formation or shrinking of the plasma column. To understand why it is possible, we proceed from the stationary heat balance at the edge homogeneous along magnetic field, i.e., from Eq. (9) with the first and second terms on the left hand side neglected:

$$
-\kappa_{\perp} d^{2} T / d x^{2}=-Q_{\mathrm{rad}}
$$

where $x$ is the distance from the LCMS toward the plasma axis and $T_{e}=T_{i}=T$ is assumed henceforth. Moreover, we take into account that with a realistic level of the impurity particle diffusion of $1 \mathrm{~m}^{2} / \mathrm{s}$ the effective cooling rate $L_{I}$ is nearly constant for the temperatures lower than a certain level $T_{\max }$ being close to the ionization energy of the impurity Li-like ions, see Fig. 2. For the sake of an analytical treatment we assume $Q_{\mathrm{rad}}(T)=Q_{0}=$ const for $T \leq T_{\max }$ and $Q_{\mathrm{rad}}=0$ for $T>T_{\max }$. The boundary condition at the LCMS, $x=0$, is given by $d T / d x=T / \delta_{T}$ with the e-folding length $\delta_{T}$ prescribed by transport processes in the scrape-off layer (SOL) beyond the LCMS. At the interface of the radiating layer with the plasma core, $x=\Delta_{\mathrm{rad}}, T=T_{\max }$ and $\kappa_{\perp} d T / d x=q_{\text {heat }}$ where the latter is determined by the heating power in the plasma core. Thus, we have three boundary conditions for the second order ordinary differential equation (18). All of them are needed because $\Delta_{\text {rad }}$ is unknown a priori.

As a result of the integration of Eq. (18) one gets the following relation for the plasma temperature at LCMS, $T_{s}$ :

$$
T_{s}^{ \pm}=\left(Q_{0} \delta_{T} \pm \sqrt{\begin{array}{c}
Q_{0}^{2} \delta_{T}^{2}+q_{\text {heat }}^{2}- \\
-2 Q_{0} \kappa_{\perp} T_{\max }
\end{array}}\right) \frac{\delta_{T}}{\kappa_{\perp}}
$$

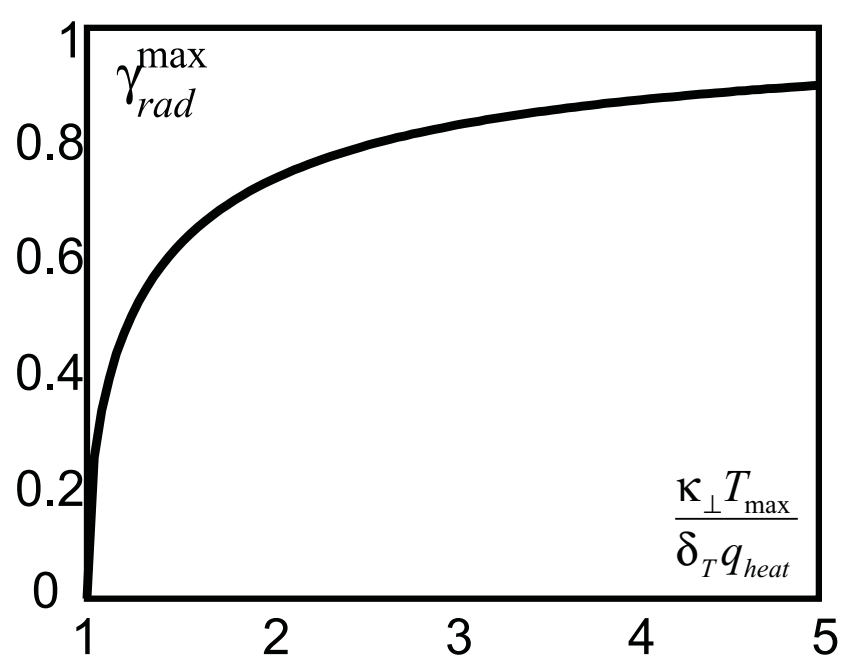

Figure 6: The variation of the maximum radiation level in stable steady states with the parameter characterizing transport, heating and impurity radiation ability.

The solution $T_{s}{ }^{-}$is unstable. This can be seen by determining $T_{s}$ from the edge power balance

$$
q_{\text {heat }}=q_{\text {loss }} \equiv q_{\mathrm{SOL}}+q_{\mathrm{rad}}
$$

where the first term in $q_{\text {loss }}$ is the heat flux $q_{S O L}=$ $\kappa_{\perp} T / \delta_{T}$ conducted by the plasma through the LCMS into the SOL and the second one, $q_{\mathrm{rad}}=\Delta_{\text {rad }} Q_{0}$, is lost with the impurity radiation. The dependence $q_{\text {loss }}$ on $T_{s}$ is shown in Fig. 5 for two different magnitudes of $Q_{0}$. Two stationary solutions exist if $Q_{0}$ is not too small and not too large. In the stationary state with $T_{s}=T_{s}^{-}$a spontaneous reduction of $T_{s}$ would lead to an increase of the energy losses due to widening of the radiating edge layer. Therefore $T_{s}$ would decrease further, i.e., an instability takes place. A similar analysis shows that the solution $T_{s}^{+}$ is stable. The maximally achievable radiation level $\gamma_{\text {rad }} \equiv \Delta_{\text {rad }} Q_{0} / q_{\text {heat }}$ in this state corresponds to the case where $T_{s}^{+}$merges with $T_{s}^{-}$when $Q_{0}$ increases up to the critical level. This provides:

$$
\gamma_{\text {rad }}^{\max }=1-\frac{\kappa_{\perp} T_{\max }}{q_{\text {heat }} \delta_{T}}+\sqrt{\left(\frac{\kappa_{\perp} T_{\max }}{q_{\text {heat }} \delta_{T}}\right)^{2}-1}
$$

Figure 6 displays the dependence of $\gamma_{\text {rad }}^{\max }$ on the parameter combination $\kappa_{\perp} T_{\max } /\left(q_{\text {heat }} \delta_{T}\right)$. One can see that with passing from Ohmic plasmas with relatively low transport (small $\kappa_{\perp}$ ) and intrinsic carbon impurity $\left(T_{\max } \approx 60 \mathrm{eV}\right)$ to additionally heated discharges with high transport (large $\kappa_{\perp}$ ) and seeding of neon $\left(T_{\max } \approx 200 \mathrm{eV}\right)$ the maximally achievable radiation level $\gamma_{\mathrm{rad}}$ without instability increases, as it takes place in the experiment [7].

When the critical level of $Q_{0}$ is exceeded, there is no anymore stationary states in the framework of the model considered above. This leads to the steadily cooling of the plasma edge. When $T_{s}$ decreases below the excitation energy of low-Z impurity ions of 
$5-10 \mathrm{eV}$, the radiating layer develops in a radiating toroidal shell which shrinks towards the plasma axis [31]. This processes can, however, be terminated: the heat flux density from the core increases because the plasma current, that is maintained constant, flows in a narrower channel. Thus, the current density and ohmic heating in the core increase. As a result a "detached plasma" state develops [27].

\section{DIVERTOR DETACHMENT CAUSED BY IM- PURITY RADIATION}

In a divertor configuration the radiation of impurities can be localized in the divertor volume, see Fig. 7, where the plasma state is essentially controlled by the recycling of charged particles and energy loss to the target plate [32]. Normally neutrals of the working gas of hydrogen isotopes are ionized very close to the plate in the "recycling zone". In that zone heat is transported by the convection of plasma particles towards the plate. By going deeper into the plasma, at a distance larger than neutrals penetrate, the intensity of the charged particle source and, thus, the plasma flux drop and in the "conduction zone" the heat is transported predominantly by the heat conduction[33].

Consider an impurity particle eroded from the divertor target and entering the SOL plasma as a neutral. Because of the difference in masses the impurity atom is ionized at a shorter distance than hydrogen ones, i.e. still in the recycling region where the plasma flows to the plate with a velocity close to the sound speed. Huge friction between the plasma flow and newly born impurity ion will drag the latter in a short time back to the plate so that it will not have any opportunity to be excited and radiate. Does it mean that there is no any chance for impurity particles to "sneak away" and enter the conduction region where the thermal force overcomes the friction? This is not the case for impurity neutrals produced sufficiently close to the lateral side of the SOL. They can escape from the plasma layer into the gas volume and return back in the conduction region of the SOL. Let us estimate the probability of such a process, $\omega_{\text {cond }}$. A neutral which has been produced at the plate at a distance $y$ from the SOL side and moves towards it with a velocity $v_{0}$ will be not ionized in the SOL with a probability of $\exp \left(-y / l_{0}\right)$, where $l_{0}=v_{0} /\left(k_{0} n_{p}\right)$ is the penetration depth with $k_{0}^{\text {ion }}$ being the ionization rate coefficient for impurity neutrals computed at the plasma temperature near the plate, $T_{p}$, and $n_{p}$ is the plasma density there. By averaging this value over the SOL width $\Delta$, we obtain:

$$
\omega_{\text {cond }}=\frac{l_{0}}{\Delta}\left[1-\exp \left(-\frac{\Delta}{l_{0}}\right)\right]
$$

For typical plasma parameters in Div-I divertor in $\operatorname{ASDEX}-\mathrm{U}[32], T_{p} \approx 10 \mathrm{eV}, n_{p} \approx 10^{20} \mathrm{~m}^{-3}, \Delta \approx$

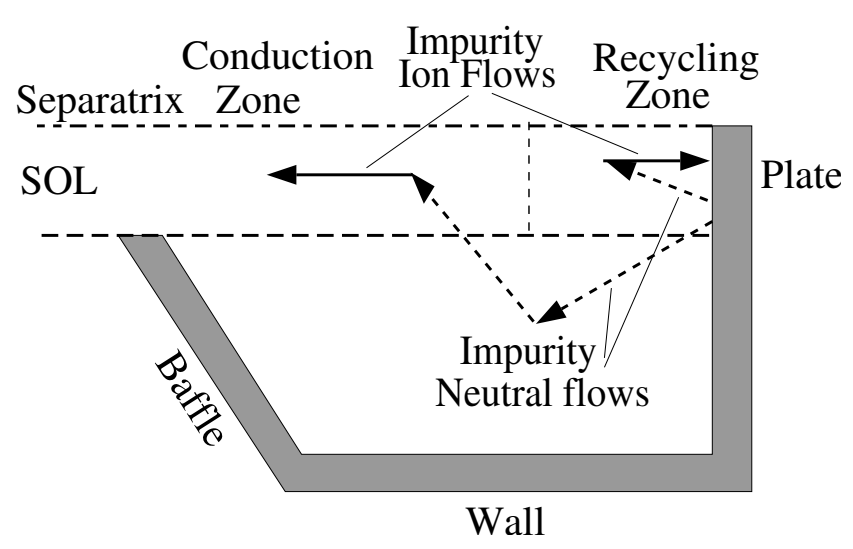

Figure 7: Geometry of the divertor volume, "recycling" and "conduction" zones in the plasma and flows of impurity neutrals and ions.

$5 \mathrm{~cm}$, one gets $\omega_{\text {cond }} \approx 0.1$ for carbon neutrals with $v_{0} \approx 10^{6} \mathrm{~cm} / \mathrm{s}$. This value is by several orders of magnitude larger than the probability of direct neutral penetration into the conduction zone of the SOL.

Another important parameter is the plasma temperature $T_{c}$ at the interface between "recycling" and "conduction" zones. This value defines the actual range of temperatures, $T_{c}<T<T_{\max }$, in which impurities radiate in the divertor SOL. To find $T_{c}$ one should consider the particle and energy transport in the recycling region. We do this in a simple onedimensional approximation by taking into account transport processes along magnetic field ( $l$-direction) only. The density of recycling hydrogen neutrals, $n_{a}$, and the plasma density are determined from the continuity equations:

$$
\begin{gathered}
d\left(-D_{a} d n_{a} / d z\right) / d z=-k_{\text {ion }} n n_{a} \\
d\left(n V_{\|}\right) / d l=k_{\text {ion }} n n_{a}
\end{gathered}
$$

where $z=-l \sin \psi$ is the distance from the plate, $\psi \approx 0.1$ the angle between the plate and magnetic field; the transport of neutrals is described in a diffusive approximation by taking into account that neutral velocities change chaotically by charge-exchanges and $D_{a}=T /\left[n m_{i}\left(k_{\mathrm{cx}}+k_{\text {ion }}\right)\right]$ is the neutral diffusivity with $k_{\mathrm{cx}}$ and $k_{\text {ion }}$ being the rate coefficients of charge-exchange and ionization of hydrogen atoms, respectively. At the plate, $l=z=0$, full recycling takes place, i.e.,

$$
-D_{a} \frac{d n_{a}}{d x}=-n V_{\|} \sin \psi
$$

The plasma parallel velocity $V_{\|}$is governed by the conservation of the parallel momentum:

$$
m_{i} n V_{\|}^{2}+2 n T=4 n_{p} T_{p}
$$

where it is taken into account that at the target the Bohm criterion, see, e.g., Ref. [20], has to be satisfied, i.e. $V_{\|}$is equal to the ion sound velocity 
$c_{s} \equiv \sqrt{2 T_{p} / m_{i}}$. In the recycling zone the radiation losses can be neglected and the plasma temperature here is governed by the conservation of the energy flux:

$$
-\kappa_{\|} d T / d l+5 n V_{\|} T=\gamma c_{s} T_{p}
$$

with $\gamma \approx 7.5$ being the so called heat transmission factor $[20]$.

The plasma temperature at the plate, $T_{p}$, is taken as a parameter and the transport equations above are integrated numerically that gives their values as at larger $|l|$ and $z$. At a certain position with the temperature $T_{c}$ the balance of the friction and thermal forces applied to impurities from the background particles is met:

$$
m_{Z} \nu_{Z i} V_{\|} \approx-\xi_{Z} d T / d l
$$

Calculations show that $T_{c} \approx T_{p}$ if $T_{p}$ exceeds $30-40$ $e V$ and exceeds $T_{p}$ by a factor of 3 if this is below $10 \mathrm{eV}$.

Consider now the conduction zone. Here the thermal force accelerates impurity ions away from the plate up to a velocity at which the friction force with background particles, being nearly at rest, is in balance with the thermal force. Thus, also in this case the balance of forces applied to impurity ions can be described by Eq. (26) but with $-V_{\|}^{Z}$ instead of $V_{\|}$. This determines the impurity velocity and density:

$$
V_{\|}^{Z} \approx \frac{\xi_{Z}}{\nu_{Z i} m_{Z}} \frac{d T}{d l}, \quad n_{I} \approx \frac{\Gamma_{I}^{c}}{V_{\|}^{Z}}
$$

where $\Gamma_{I}^{c}$ is the influx density of impurity neutrals into the conduction region averaged over the SOL width. This value is governed both by the impurity source due to erosion of the plate and by the conductance of the recycling region for neutrals. The former one is determined by the density of the plasma flux to the plate and the erosion coefficient $Y_{I}$. As a result we get:

$$
\Gamma_{I}^{c} \approx n_{p} c_{s} \cdot Y_{I} \cdot \omega_{\text {cond }}
$$

In the conduction part of the SOL the heat balance equation should take into account the energy losses with radiation,

$$
\frac{d}{d l}\left(-\kappa_{\|} \frac{d T}{d l}\right)=-n n_{I} L_{I}
$$

Since the plasma velocity here is much smaller than the ion sound speed, Eq. (24) results in the pressure balance $n T=2 n_{p} T_{p}=P_{s}$ with $P_{s}$ being the plasma pressure at the separatrix. With the definitions of $\nu_{Z i}$ and $\kappa_{\|}[21]$ and the $n_{I}$ found above, we obtain:

$$
\frac{d}{d l}\left(\kappa_{\|} \frac{d T}{d l}\right) \approx \sqrt{\frac{m_{i}}{m_{e}}} P_{s}^{2} L_{I} \Gamma_{I}^{c}\left(T \kappa_{\|} \frac{d T}{d l}\right)^{-1}
$$

This equation, multiplied by $3 \kappa_{\|}^{2} \frac{d T}{d l} d T$ and integrated over $T$ from $T_{c}$ to $T_{\max }$, provides an equation for the plasma temperature at the target plate:

$$
F_{p}+F_{r} \approx R
$$

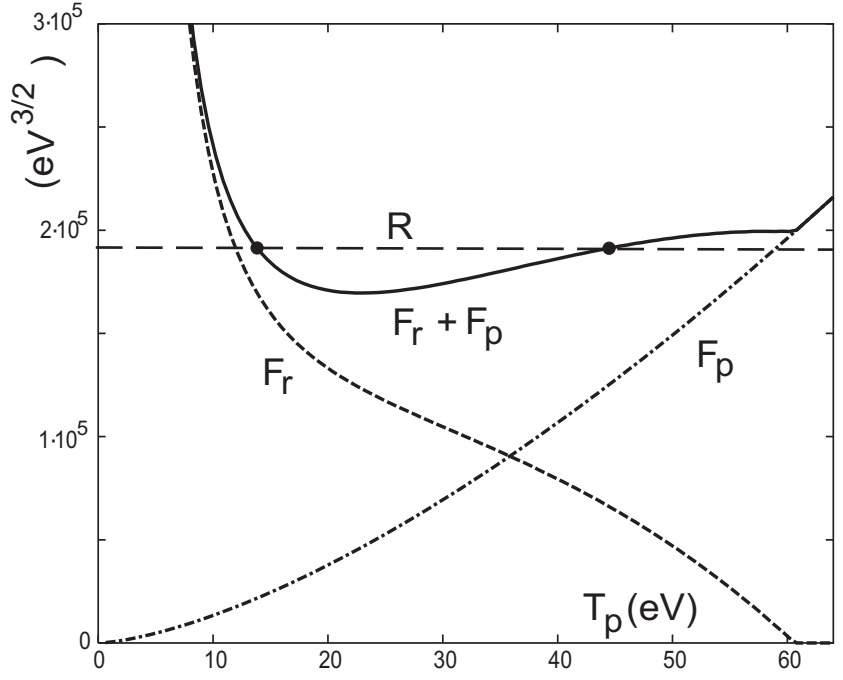

Figure 8: Functions $F_{p}\left(T_{p}\right)$ and $F_{p}\left(T_{p}\right)$ and their sum vs the plasma temperature at the target plate.

where $F_{p}=\gamma^{3} T_{p}^{1.5}$ and

$$
F_{r}=2.4 Y_{I} \omega_{\text {cond }} \sqrt{\frac{m_{i}}{m_{e}}} m_{i} L_{0} \frac{\kappa_{\|}\left(T_{\max }\right)-\kappa_{\|}\left(T_{c}\right)}{\sqrt{T_{p}}}
$$

and their sum are displayed in Fig. 8 as functions of $T_{p}$ for deuterium SOL plasma in contact with divertor plates of carbon. The erosion coefficient $Y_{I}=0.02$ was assumed independent of $T_{p}$ since at low temperatures physical sputtering [18] of carbon is replaced by chemical erosion with roughly constant $Y_{I}$ [19].

The right hand side of Eq. (31),

$$
R \equiv\left(q_{\text {heat }}^{\|} \sqrt{2 m_{i}} / P_{s}\right)^{3}
$$

with $q_{\text {heat }}^{\|}$being the density of the parallel heat flux into the divertor, is independent of $T_{p}$ but controlled by global plasma parameters such as the heating power $W_{\text {heat }}$ and the mean density $\langle n\rangle$. For a Bohmlike perpendicular transport in the SOL [20]:

$$
R \sim W_{\text {heat }}^{2.7} /\langle n\rangle^{4.7}
$$

Thus, as in the case of the edge radiating layer in the confinement region with perpendicular energy transport, see section IV and Fig. 5, stationary states in divertor exist if $R$ is large enough, i.e. for sufficiently strong heating and not too high plasma density. If the former is fixed and the density increases above the critical level there are no steady state, the plasma near the target plate cools down due to impurity radiation, detachment from the plate and $\mathrm{X}$-point MARFE should develop. The used system of transport equations fails to describe this evolution because many processes important at low temperatures are not taken into consideration. In particular, when $T_{p}$ becomes noticeably less than the ionization potential of hydrogen, neutrals recycling from the plate freely 
escape into the gas surrounding the plasma layer. These particles return later into the SOL through its lateral side and contribute significantly to momentum and heat dissipation [33]. Additionally, diverse channels for recombination of charged particles can influence all balances and govern the plasma state in a detached divertor.

\section{REFERENCES}

1. D.M. MEADE, Nucl. Fusion 14, 289 (1974).

2. R.V. JENSEN, et al., Nucl. Fusion 17, 1187 (1977).

3. V.A. VERSHKOV and S.V. MIRNOV, Nucl. Fusion 14, 383 (1974).

4. R.J. HAWRYLUK, et al., Nucl. Fusion 19, 1307 (1979).

5. D.E. POST, et al., Atomic Data and Nuclear Data Tables 20, 397 (1977).

6. Y. SHIMOMURA, Nucl. Fusion 17, 626 (1977).

7. U. SAMM, et al., Plasma Phys. Contr. Fusion 35, B167 (1993).

8. R.S. GRANETZ, et al., Nucl. Fusion 47, 1086 (2007).

9. G.M. MCCRACKEN, et al., J. Nucl. Mater. 145-147, 181 (1987).

10. U. SAMM, et al., J. Nucl. Mater. 176-177, 273 (1990).

11. J. WESSON, Tokamaks, third Edition, Clarendon Press, Oxford (2004).

12. J.L. TERRY, et al., Bul. Am. Phys. Soc. 26, 886 (1981).

13. F. ALlADiO, et al., Phys. Lett. 90A, 405 (1982).

14. D.R. BAKER, et al., Nucl. Fusion 22, 807 (1982).

15. A. LOARTE, et al., Nucl. Fusion 38, 331 (1998).

16. E.A. LASARUS, et al., J. Nucl. Mater. 121, 61 (1984).

17. J. ONGENA, et al., Physica Scripta 52, 449 (1995).

18. W. ECKSTEIN, et al., Atomic and PlasmaMaterial interaction data for fusion (Suppl. to Nucl.Fusion) 1, 51 (1991).

19. A.A. HAASZ, et al., Atomic and PlasmaMaterial interaction data for fusion (Suppl. to Nucl.Fusion) 7A, 9 (1998).
20. P.C. STANGEBY The Plasma Boundary of Magnetic Fusion Devices, IoP Publishing, Bristol, (2000).

21. S.I. BRAGINSKII in Reviews of Plasma Physics edited by Leontovich M A, New York, Consultants Bureau, 1, 205 (1965).

22. S. CHAPMAN Proc. Phys. Soc., London, Sect. A, 72, 353 (1958).

23. S.P. HIRSHMAN and D.J. SIGMAR, Nucl. Fusion 21, 1079 (1981).

24. J. WEILAND Collective Modes in Inhomogeneous Plasmas, Kinetic and Advanced Fluid Theory, IOP Publishing, Bristol (2000)

25. M.Z. TOKAR and F.A. KELLY, Phys. Plasmas 10, 4378 (2003).

26. D.E. POST, J. Nucl. Mater. 220-222, 143 (1995).

27. M.Z. TOKAR, Plasma Phys. Control. Fusion 36, 1819 (1994).

28. M. KOLTUNOV and M.Z. TOKAR Plasma Phys. Control. Fusion 54, 025003 (2012).

29. M.Z. TOKAR and M. KOLTUNOV, Phys. Rev. E 85, 046412 (2012).

30. M.Z. TOKAR, et al., J. Nucl. Mater 266-269, 958 (1999).

31. U. SAMM, et al., Properties of "detached" plasmas, IPP Rep. Jül-2123, 1987.

32. U. WENZEL, et al., Plasma Phys. Control. Fusion, 41, 801 (1999).

33. A.V. NEDOSPASOV and M.Z. TOKAR, in Reviews of Plasma Physics, edited by Kadomtsev B.B., Consultants Bureau 18, 77 (1993). 